\title{
Inhibition of adipogenic differentiation by myostatin is alleviated by arginine supplementation in porcine-muscle-derived mesenchymal stem cells
}

\author{
LEI HuLong, YU Bing, YANG XueRong, LIU ZeHui, HUANG ZhiQing, MAO XiangBing, \\ TIAN Gang, HE Jun, HAN GuoQuan, CHEN Hong, MAO Qian \& CHEN DaiWen* \\ Key Laboratory for Animal Disease-Resistance Nutrition of Ministry of Education of China, Animal Nutrition Institute, \\ Sichuan Agricultural University, Ya'an 625014, China
}

Received May 12, 2011; accepted August 30, 2011

\begin{abstract}
Porcine mesenchymal stem cells in postnatal muscle have been demonstrated to differentiate into adipocytes. This increases adipocyte number and lipid accumulation, and is thought to be the origin of intramuscular fat. In this study, the effects of myostatin and arginine on adipogenic differentiation in mesenchymal stem cells derived from porcine muscle (pMDSCs) were investigated in vitro. Intracellular triglyceride levels were reduced by exogenous myostatin and increased by arginine supplementation or myostatin antibody $(P<0.01)$. The inhibition of lipid accumulation by myostatin in pMDSCs was alleviated by arginine supplementation $(P<0.01)$. Expression patterns of adipogenic transcription factors showed that exogenous myostatin suppressed $P P A R \gamma 2$ and $a P 2$ expression $(P<0.01)$, while supplemental arginine or myostatin antibody promoted $A D D 1$ expression $(P<0.01)$. Furthermore, compared with the addition of either myostatin protein or antibody alone, ADDI and $P P A R \delta$ expression were promoted by the combination of arginine and myostatin $(P<0.01)$, and arginine combined with myostatin antibody promoted the expression of $A D D 1, P P A R \delta, C / E B P \alpha, P P A R \gamma 2$ and $L P L$ in pMDSCs $(P<0.05)$. These results suggest that myostatin inhibits adipogenesis in pMDSCs, and that this can be alleviated by arginine supplementation, at least in part, through promoting $A D D 1$ and $P P A R \delta$ expression.
\end{abstract}

myostatin, arginine, adipogenic differentiation, mesenchymal stem cells, porcine

Citation: Lei H L, Yu B, Yang X R, et al. Inhibition of adipogenic differentiation by myostatin is alleviated by arginine supplementation in porcine-muscle-derived mesenchymal stem cells. Sci China Life Sci, 2011, 54: 908-916, doi: 10.1007/s11427-011-4227-1

Over the last few decades, the growth performance of domestic animals has been improved by genetic selection, comfortable circumstances, and optimal nutrition, which has led to poor meat quality [1]. Intramuscular fat (IMF) is one of the main factors affecting meat quality, and has attracted increasing attention in animal production [2,3]. Adipose tissue is composed of numerous adipocytes containing large numbers of lipid droplets. The IMF content thus depends on the adipocyte number and lipid accumulation. Satellite cells or mesenchymal stem cells residing in postnatal muscle

*Corresponding author (email: dwchen@ sicau.edu.cn) have been shown to differentiate into adipocytes [4-7], and are believed to be the source of IMF [8].

Arginine is an essential amino acid for pregnant sows and newborn piglets, and is required for fetal and neonatal growth and development [9-11]. Arginine supplementation has been reported to increase the IMF content of the longissimus dorsi, while decreasing carcass fat content in growing-finishing pigs [12]. It has been suggested that arginine modulates fat deposition in skeletal muscle and subcutaneous fat in different ways. Myostatin, also known as GDF-8, is a negative regulator of skeletal muscle growth and development $[13,14]$. Myostatin was recently reported to in- 
hibit myogenesis and promote adipogenesis [15], and was able to substitute for dexamethasone in inducing adipogenic differentiation of mouse mesenchymal stem cells (MSCs) in vitro [16]. Nevertheless, inconsistent results have been reported in primary human bone marrow MSCs [17]. Despite this, it has been suggested that myostatin is involved in fat deposition and modulation of adipogenic differentiation. Although the roles of myostatin in skeletal muscle growth regulation have been widely recognized, its effects on adipogenic differentiation are poorly understood. In this study, we therefore focused on the effects of myostatin on adipogenesis in porcine-muscle-derived mesenchymal stem cells (pMDSCs), and the modulation of this activity by arginine. Furthermore, the involvement of several adipogenic transcription factors, including $A D D 1, C / E B P \alpha, P P A R \gamma$ and $P P A R \delta$, was investigated to improve our understanding of the molecular mechanisms of the process.

\section{Materials and methods}

\subsection{Animals}

Crossed Duroc $\times$ Landrace $\times$ Yorkshire (DLY) growing pigs weighing about $35 \mathrm{~kg}$ were used. The animals were housed in the Experimental Farm of Animal Nutrition Institute, Sichuan Agricultural University. All animal handling was conducted according to Institutional Guidelines after acquiring permission from Sichuan Agricultural University Institutional Animal Care and Use Committee.

\subsection{Isolation and culture of pMDSCs}

pMDSCs were harvested as described previously [18], with modifications. Briefly, longissimus dorsi muscles were dissected from DLY growing pigs and the vascular tissue and fascia were removed. Samples were washed three times in Hank's balanced salt solution (HBSS), minced into small pieces and digested with $0.2 \%$ type II collagenase (Gibco, Grand Island, NY) at $37^{\circ} \mathrm{C}$ for $40-60 \mathrm{~min}$. After digestion, suspensions were washed twice in HBSS and centrifuged at $1500 \mathrm{r} \mathrm{min}^{-1}$ for $5 \mathrm{~min}$. Pellets were suspended in DMEM/ F12 (1:1) (Dulbecco's Modified Eagle Media: Nutrient Mixture F-12 (1:1), Gibco) and passed through 40- $\mu \mathrm{m}$ cell strainers (Becton Dickinson) to yield single-cell suspensions.

Nucleated cells were centrifuged and resuspended in growth medium, DMEM/F12 (1:1) (Gibco) containing 10\% fetal bovine serum (MSC qualified, Gibco), $100 \mathrm{U} \mathrm{mL}^{-1}$ penicillin and $100 \mu \mathrm{g} \mathrm{mL} \mathrm{m}^{-1}$ streptomycin (Gibco). Cells were plated in 35-mm tissue culture treated dishes (Corning, Lowell, MA) and incubated at $37^{\circ} \mathrm{C}$. MSCs were isolated primarily based on their adhesion characteristics. Suspensions with nonadherent cells were transferred into 100-mm dishes or T75 tissue culture treated flasks (Corning) after $15 \mathrm{~min}$ incubation, to exclude contaminating macrophages, most of which would be attached to the culture dish. Cells were cultured at $37^{\circ} \mathrm{C}$ in a saturated humidity, $5 \% \mathrm{CO}_{2}$ tissue-culture incubator as passage 0 . Forty-eight hours after initial plating, the cells were washed twice with HBSS to remove nonadherent cells, cultured in fresh growth medium, and half the medium was replaced every three days. Once the cells reached $70 \%-80 \%$ confluence, they were detached with $0.25 \%$ trypsin- $0.038 \%$ EDTA (Gibco) and reseeded in fresh medium, as above. MSCs were extracted based on their adherent characteristics and purified during serial passages.

The fibroblast-like spindle morphology of cells derived from porcine muscle was assessed under a microscope (Axiosvert 2000 microscope equipped with AxioCam MRc 5 camera and AxioVision Rel. 4.6 software; Carl Zeiss, Jena, Germany). For further characterization, the immunophenotype was determined by immunofluorescence histochemistry and fluorescence-activated cell sorting (FACS) using the antibodies described below. Multipotency of cells was confirmed by multiple-lineage induction and characterization of corresponding mesenchymal cells. Cells were expanded in culture up to eighth passage, and only early passage cells (passages 3-8) were used.

\subsection{Immunofluorescence with multiple labeling}

Cells were labeled and observed as previously described [19] with modifications. Exponentially-grown cells were washed twice with phosphate-buffered saline (PBS), fixed in $4 \%$ paraformaldehyde for $20 \mathrm{~min}$ and permeabilized in $0.5 \%$ Triton X-100 in PBS for 30 min with gentle rocking. After fixation and permeabilization, cells were rinsed $(3 \times 5 \mathrm{~min})$ in PBS, blocked with 5\% normal donkey serum in PBS for $30 \mathrm{~min}$, incubated with purified rat antibodies to porcine CD90 in PBS for $2 \mathrm{~h}$, and incubated with fluorescein isothiocyanate (FITC)-conjugated affinity-purified $\mathrm{F}(\mathrm{ab}) 2$ fragment donkey anti-rat $\mathrm{IgG}(\mathrm{H}+\mathrm{L})(\min \times$ bovine, chicken, goat, guinea pig, Syrian hamster, horse, human, mouse, rabbit and sheep serum proteins) in PBS for $30 \mathrm{~min}$. For multiple labeling, the above procedure was followed, and cells were incubated with purified mouse antibodies to porcine CD29, and incubated with rhodamine (TRITC)-conjugated affinity-purified $\mathrm{F}\left(\mathrm{ab}^{\prime}\right) 2$ fragment donkey anti-mouse $\operatorname{IgG}(\mathrm{H}+\mathrm{L})$ (min $\times$ bovine, chicken, goat, guinea pig, Syrian hamster, horse, human, rabbit, rat and sheep serum proteins) in PBS. Cells were nuclear stained with 4',6-diamidino-2-phenylindole (DAPI) for $20 \mathrm{~min}$. Thorough washing was performed after each step, including after blocking. Glycerol $50 \%$ in PBS was added to protect fluorescence followed by immediate visualization using an Axiosvert 2000 microscope equipped with an AxioCam MRc 5 camera and AxioVision Rel. 4.6 software (Carl Zeiss). Images were collected sequentially (ZVI files), converted to JPG files, and contrast-stretched without further processing. Rat monoclonal 
IgG CD90 and mouse monoclonal IgG CD29 were used as primary antibodies (BD Biosciences), diluted at 1:100. Donkey anti-rat IgG conjugated with FITC or donkey anti-mouse IgG conjugated with TRITC were used as secondary antibodies for immunofluorescence (multiple-labeling grade; Jackson Immunoresearch Laboratories, West Grove, PA), at 1:200 dilution. Negative control cells were incubated with mouse or rat IgG antibody (BD Biosciences) under the same conditions.

\subsection{Flow cytometry and phenotypic analysis}

Standard flow cytometry techniques were used to determine the cell surface epitope profile (CD31, CD34, CD45, CD29, CD49f, and CD90). Briefly, prior to antibody labeling, the cells were washed twice with PBS. Cells were incubated with purified mouse antibodies to porcine CD29 (BD Biosciences) or CD45 (Santa Cruz Biotechnology, Delaware Avenue, CA) at 1:100 dilution for $30 \mathrm{~min}$, then incubated with allophycocyanin (APC)-conjugated affinity-purified $\mathrm{F}\left(\mathrm{ab}^{\prime}\right) 2$ fragment donkey anti-mouse $\mathrm{IgG}(\mathrm{H}+\mathrm{L})$ (min×bovine, chicken, goat, guinea pig, Syrian hamster, horse, human, rabbit, rat and sheep serum proteins) (multiple-labeling grade; Jackson Immunoresearch Laboratories) at a 1:200 dilution in PBS. Cells were incubated with phycoerythrin (PE)-conjugated mouse monoclonal antibodies to porcine CD31 (BD Biosciences). After labeling with APC or PE, cells were incubated with FITC-conjugated rat monoclonal antibodies to porcine CD34, CD49f, or CD90 (all antibodies were purchased from BD Biosciences, San Diego, CA) for multiple labeling. The isotype (negative) controls used in this study were subjected to the same conditions to evaluate the levels of nonspecific binding. All cell suspensions were washed twice with PBS and resuspended in PBS for analysis on a flow cytometer (FACSCalibur; BD Biosciences). At least 100000 labeled cells were collected and the data were analyzed using FCS Express Version 3.0 (De Novo Software, Los Angeles, CA).

\subsection{Multilineage differentiation assays}

Cells were plated in growth medium at a density of $5 \times 10^{3}$ $\mathrm{cm}^{-2}$ for $48 \mathrm{~h}$ to reach $70 \%-80 \%$ confluency. For myogenic differentiation, cells were incubated in DMEM-LG (Dulbecco's Modified Eagle Media, low glucose, Gibco) supplemented with $10 \mu \mathrm{mol} \mathrm{L}{ }^{-1}$ 5-azacytidine, $2 \%$ horse serum and antibiotics at $37^{\circ} \mathrm{C}$ for the initial $24-\mathrm{h}$ period, then washed twice with PBS and maintained in the same conditions without 5-azacytidine for up to four weeks. Desmin expressed in skeletal muscle plays an important role in skeletal muscle cell differentiation and was detected with purified mouse antibody to porcine desmin (Santa Cruz) and TRITC-conjugated donkey anti-mouse IgG $(\mathrm{H}+\mathrm{L})$ (Jackson Immunoresearch Laboratories) by immunohistochemistry, as described above, and multinucleated myotubes were ob- served under a phase contrast microscope (Axiovert 2000, Carl Zeiss). For adipogenic differentiation, cells were incubated in medium containing high-glucose DMEM (DMEM-HG) with 10\% FBS (Gibco), $1 \mu \mathrm{mol} \mathrm{L} \mathrm{L}^{-1}$ dexamethasone, $10 \mathrm{mg} \mathrm{L}^{-1}$ insulin, $0.5 \mathrm{mmol} \mathrm{L}^{-1}$ 3-isobutyl-1methylxanthine, $100 \mu \mathrm{mol} \mathrm{L}{ }^{-1}$ indomethacin, $100 \mathrm{U} \mathrm{mL}^{-1}$ penicillin and $100 \mu \mathrm{g} \mathrm{mL}^{-1}$ streptomycin (Gibco) (DIM). After $14 \mathrm{~d}$ induction, formation of lipid vacuoles in the cells was visualized by Oil Red $\mathrm{O}$ staining according to published protocols [20]. For osteogenic differentiation, cells were cultured in low-glucose DMEM (DMEM-LG) supplemented with $10 \%$ FBS (Gibco), $100 \mathrm{nmol} \mathrm{L}^{-1}$ dexamethasone, $10 \mathrm{mmol} \mathrm{L}{ }^{-1} \beta$-glycerophosphate, $50 \mu \mathrm{g} \mathrm{L}^{-1}$ ascorbic acid, $100 \mathrm{U} \mathrm{mL}^{-1}$ penicillin and $100 \mu \mathrm{g} \mathrm{mL}^{-1}$ streptomycin (Gibco). On day 21, cultures were stained for alkaline phosphatase using the calcium-cobalt method and extracellular matrix mineralization was assessed by von Kossa staining for four weeks. Medium was changed every 3-4 days. All the reagents were from Sigma (Sigma, Saint Louis, MO), unless otherwise specified.

\subsection{Adipogenic differentiation and modulation}

Cells were plated in growth medium at a density of $5 \times 10^{3}$ $\mathrm{cm}^{-2}$ for $48 \mathrm{~h}$ to reach $70 \%-80 \%$ confluency. To exclude disturbances caused by the presence of arginine in the basal DMEM medium, arginine-deficient DMEM-HG was customized and provided by Sunrise Science (San Diego, CA). Cells were exposed to either $50 \mu \mathrm{g} \mathrm{mL}^{-1}$ arginine (Sigma), $100 \mathrm{ng} \mathrm{mL}{ }^{-1}$ recombinant myostatin (PeproTech, Rocky Hill, NJ), $4 \mu \mathrm{g} \mathrm{mL} \mathrm{m}^{-1}$ monoclonal rat antibody to human myostatin (R\&D Systems, McKinley Place, NE), arginine combined with recombinant myostatin, or arginine combined with the monoclonal antibody to myostatin, in addition to DIM adipogenic induction medium. Negative and differentiation control group cells were exposed to DMEM-HG (arginine-deficient) supplemented with $10 \%$ FBS, $100 \mathrm{U} \mathrm{mL}^{-1}$ penicillin and $100 \mu \mathrm{g} \mathrm{mL}^{-1}$ streptomycin, or DIM, respectively. Cells were cultured at $37^{\circ} \mathrm{C}$, in a saturated humidity, $5 \% \mathrm{CO}_{2}$ tissue-culture incubator, and the medium was replaced with corresponding fresh medium every three days. The expression of adipogenic transcription factors on day 2 , and the accumulation of intracellular triglycerides after $14 \mathrm{~d}$ induction were measured by real-time quantitative polymerase chain reaction (PCR) and fluorometric assay.

\subsection{Adipogenesis assay}

On day 14, intracellular triglyceride accumulation, in the form of lipid droplets, was determined using an Adipogenesis Assay Kit (BioVision, Mountain View, CA), according to the manufacturer's instructions. This assay can detect triglycerides in $\leqslant 1000$ differentiated adipocytes with high sensitivity. A fluorometric assay at excitation and emission 
wavelengths of 535 and $587 \mathrm{~nm}$, respectively, was performed using a Varioskan Flash (Thermo Fisher Scientific, Waltham, MA). Protein concentrations were detected with BCA Protein Assay Reagent (Pierce, Rockford, IL) and the triglyceride content was corrected to protein content.

\subsection{RNA isolation and reverse transcription-PCR}

Total RNA was extracted from adherent cultured cells using RNAiso Plus reagent (Takara, Dalian, China), according to the manufacturer's instructions. cDNA synthesis was performed for $15 \mathrm{~min}$ at $37^{\circ} \mathrm{C}$ and $5 \mathrm{~s}$ at $85^{\circ} \mathrm{C}$, using $2 \mu \mathrm{g}$ total RNA as a template, $5 \times$ primer script, random 6-mer, primer script RT enzyme Mix, oligo dT primer $\left(50 \mu \mathrm{mol} \mathrm{L}{ }^{-1}\right)$ (Takara). Total RNA and cDNA was stored at $-70^{\circ} \mathrm{C}$ until use.

\subsection{Real-time quantitative PCR}

Real-time quantitative PCR was performed in a CFX96 Real-Time System (Bio-Rad, Berkeley, CA) using SsoFast EvaGreen Supermix (Bio-Rad) in a final volume of $20 \mu \mathrm{L}$. The thermal cycling conditions were $95^{\circ} \mathrm{C}$ for $30 \mathrm{~s}$ followed by 40 cycles of $95^{\circ} \mathrm{C}$ for $10 \mathrm{~s}$ and $60-62^{\circ} \mathrm{C}$ for $10 \mathrm{~s}$. Each primer pair designed with Primer 3.0 was blasted against expected published sequences to confirm specificity, and yielded a single peak in the melting curve and a single band of the expected size in agarose gel electrophoresis. The gene-specific primers used were synthesized by Invitrogen and are listed in Table 1. Standard curves were established to measure the amplification efficiency of all target and housekeeping genes, which ranged from $102.4 \%$ to $119.9 \%$. The relative expression of the target gene was calculated using the formula $2^{-\left(\Delta \Delta C_{\mathrm{t}}\right)}$, where $\Delta \Delta C_{\mathrm{t}}$ is the difference between the sample $\Delta C_{\mathrm{t}}$ and the control (untreated) $\Delta C_{\mathrm{t}}, \Delta C_{\mathrm{t}}$ is the difference in $C_{\mathrm{t}}$ between the gene of interest and the geometric average of the reference genes, and $C_{\mathrm{t}}$ is the cycle at which the threshold is crossed. All samples were run at least in duplicate. Target gene expression was normalized to the commonly used reference genes, $A C T B, G A P D H$ and 18s rRNA, as described previously [21].

\subsection{Statistical analyses}

Statistical analyses were performed using SAS software (Release 8.1; SAS Inst. Inc.). After confirming normal distribution on histograms, data were analyzed by one-way ANOVA using the generalized linear model procedure. Data were presented as mean \pm standard deviation. Differences among means were tested by the least significant difference method and $P<0.05$ was considered to be statistically significant.

Table 1 Primer pairs used for real-time quantitative $\mathrm{PCR}^{\mathrm{a})}$

\begin{tabular}{|c|c|c|c|c|c|}
\hline No. & Gene name & GenBank ID & Primer sequence & Product size & $\operatorname{Tm}\left({ }^{\circ} \mathrm{C}\right)$ \\
\hline \multirow[b]{2}{*}{1} & \multirow[b]{2}{*}{$A C T B$} & \multirow{2}{*}{ XM_003124280.1 } & F: CATCGTCCACCGCAAAT & \multirow{2}{*}{210} & \multirow[b]{2}{*}{60} \\
\hline & & & R: TGTCACCTTCACCGTTCC & & \\
\hline \multirow{2}{*}{2} & \multirow{2}{*}{ GAPDH } & \multirow{2}{*}{ XM_003126534.1 } & F: GGCTACAGCAACAGGGTG & \multirow{2}{*}{188} & \multirow{2}{*}{60} \\
\hline & & & R: GGGTCTGGGATGGAAACT & & \\
\hline \multirow{2}{*}{3} & \multirow{2}{*}{$18 s$ rRNA } & \multirow{2}{*}{ NR_002170.3 } & F:TTAGAGTGTTCAAAGCAGGCCCGA & \multirow{2}{*}{198} & \multirow{2}{*}{60} \\
\hline & & & R: TCTTGGCAAATGCTTTCGCTCTGG & & \\
\hline 4 & $C / E B P \alpha$ & AF103944 & R: CGTGGAGACTCAACAGAAGG & 95 & 60 \\
\hline \multirow{2}{*}{5} & \multirow{2}{*}{$P P A R \gamma 2$} & \multirow{2}{*}{ NM_214379.1 } & F:AATTAGATGACAGCGACCTGGCGA & \multirow{2}{*}{102} & \multirow{2}{*}{60} \\
\hline & & & R: TGTCTTGAATGTCCTCGATGGGCT & & \\
\hline \multirow[b]{2}{*}{6} & \multirow[b]{2}{*}{$L P L$} & \multirow{2}{*}{ NM_214286.1 } & F: ACCGTTGCAACAACTTGGGCTATG & \multirow[b]{2}{*}{97} & \multirow[b]{2}{*}{60} \\
\hline & & & R:ACTTTGTAGGGCATCTGAGCACGA & & \\
\hline \multirow{2}{*}{7} & \multirow{2}{*}{$a P 2$} & \multirow{2}{*}{ NM_001002817.1 } & F: GGCCAAACCCAACCTGA & \multirow{2}{*}{167} & \multirow{2}{*}{60} \\
\hline & & & R: GGGCGCCTCCATCTAAG & & \\
\hline 8 & $A D D I$ & AY49680/ & R: GGTCCTTCAGAGACTTGCTT & 95 & 60 \\
\hline 0 & & NIM214152 & F: GCCTCCATCGTCAATAAG & 166 & 60 \\
\hline 9 & PРARठ & NM214152.1 & R: GAGCCAGGTCACTATCATC & 166 & 60 \\
\hline 10 & CATA-? & NM213870 & F: CGACAACCACCACCTTATG & 122 & 60 \\
\hline 10 & GAIA-Z & NIV12138/9.1 & R: CTGGATGCCTTCCTTCTTC & 122 & 00 \\
\hline 11 & $C A T A-3$ & NM0010445671 & F: CAAACCACAACTACGACTCTC & 125 & 60 \\
\hline 11 & GAIA-3 & NIVI00104456/.1 & R: TGGATGCCTTCCTTCTTCA & 125 & 60 \\
\hline
\end{tabular}

a) Abbreviations: ACTB, $\beta$-actin; GAPDH, glyceraldehyde 3-phosphate dehydrogenase; $18 \mathrm{~s}$ rRNA, $18 \mathrm{~s}$ ribosomal ribonucleic acid; $P P A R \gamma 2$, peroxisome proliferator-activated receptor $\gamma 2 ; L P L$, lipoprotein lipase; $a P 2$, fatty acid-binding protein adipocyte P2; ADD1, adipocyte determination and differentiation factor 1; C/EBP $\alpha$, CCAAT/enhancer binding protein $\alpha$; PPAR $\delta$, peroxisome proliferator-activated receptor $\delta$; GATA-2, GATA binding protein 2 ; GATA-3, GATA binding protein 3 ; Tm, annealing temperature. 


\section{Results}

\subsection{Characterization of adherent cells from porcine muscle}

Cells were characterized by their adherence to plastic and by their fibroblast-like spindle morphology. Adherent cells from porcine longissimus dorsi were initially heterogeneous (Figure 1A and B), but the cellular morphology became homogeneous during subculturing (Figure 1C and D). No abnormal morphology was noted up to passage 8 . For further characterization, the immunophenotype was determined by immunofluorescence histochemistry and FACS using the antibodies described. Their non-hematopoietic and non-endothelial origin (CD45-, CD31-, CD34-negative) (Figure 1I), MSC marker expression (CD29 and CD90) (Figure 1E-I) and their capabilities of differentiating into myoblasts (Figure 2A-D), preadipocytes (Figure 2E-H), and osteoblasts (Figure 2I-L) were confirmed. Their cellular morphology, expression of surface antigens, and multipotency all indicated that the cells investigated in this study were pMDSCs.

\subsection{Adipogenesis assay}

As shown in Figure 3, compared with DMEM-HG (negative control), triglyceride levels were increased in cells after 14 days adipogenic induction $(P<0.01)$. Intracellular triglyceride levels were reduced under adipogenic differentiation-induction conditions (DIM) by myostatin protein in addition to DIM $(P<0.01)$, and were increased by supplemental arginine or myostatin antibody. Lipid accumulation was not promoted by a combination of arginine and myostatin antibody, compared to cells exposed to myostatin antibody alone, but the inhibition of lipid accumulation in pMDSCs by myostatin was alleviated by arginine supplementation $(P<0.01)$. These results suggest that exogenous myostatin inhibited adipogenesis, but this effect could be alleviated by arginine supplementation.
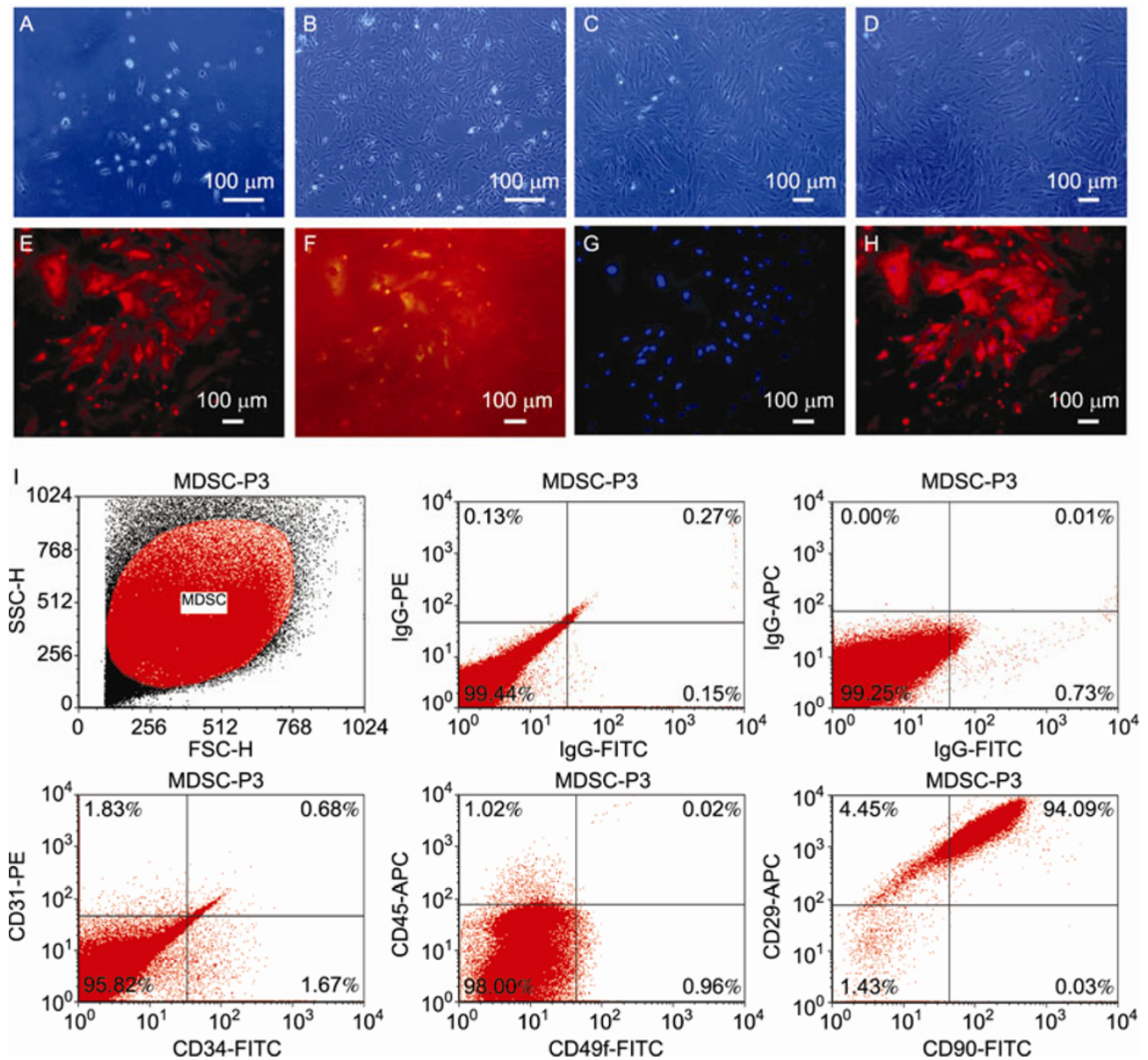

Figure 1 Morphology and phenotypic characterization of pMDSCs. Cellular morphology of pMDSCs was observed by microscopy on day 3 (A), or at confluence phase at passage 0 (B), 1 (C), and 3 (D). Immunophenotype was determined by immunofluorescence histochemistry and flow cytometry. CD29 (E) and CD90 (F) were stained with rhodamine (TRITC, red) or fluorescein isothiocyanate (FITC, green), respectively. Nucleoli (G) were stained with 4',6-diamidino-2-phenylindole (DAPI, blue), and the confocal images (H) were merged with SPOT 4.6 software. At least 100000 labeled cells were collected and the data was analyzed with FCS Express Version 3.0 (De Novo Software) (I). 



Figure 2 Multiple differentiation potentials of pMDSCs. A-D, Morphological changes and multinucleated myotube formation were observed under a microscope. Desmin expression was confirmed by immunofluorescence, and desmin was stained with rhodamine (TRITC, red) after 28 days of myogenic differentiation induction. E-H, Lipid accumulation after adipogenic induction was confirmed by Oil red O staining. Nucleoli were stained with 4',6-diamidino-2-phenylindole (DAPI, blue) and the confocal images (D and H) were merged with SPOT 4.6 software (I-L). The osteogenic phenotype of the cells was identified after four weeks of osteogenic induction. Compared with the controls (I and K), increasing alkaline phosphatase activity (J) and mineralized matrix (L) were demonstrated by Ca-Co stain and von Kossa stain, respectively.

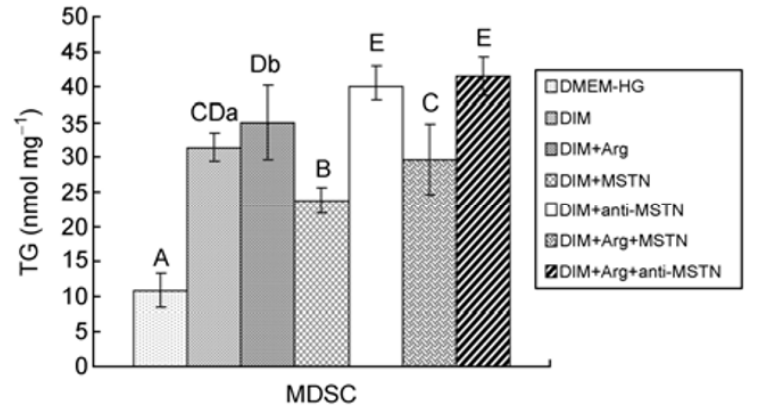

Figure 3 Intracellular triglyceride contents of cells from porcine muscle after 14 days of adipogenic induction. DIM+Arg/MSTN/anti-MSTN, DIM medium supplemented with either $50 \mu \mathrm{g} \mathrm{mL}^{-1}$ arginine, $100 \mathrm{ng} \mathrm{mL}^{-1}$ recombinant myostatin, or $4 \mu \mathrm{g} \mathrm{mL}^{-1}$ monoclonal antibody to myostatin; DIM+Arg+MSTN, DIM medium supplemented with $50 \mu \mathrm{g} \mathrm{mL}^{-1}$ arginine and $100 \mathrm{ng} \mathrm{mL} \mathrm{m}^{-1}$ recombinant myostatin; DIM+Arg+anti-MSTN, DIM medium supplemented with $50 \mu \mathrm{g} \mathrm{mL} L^{-1}$ arginine and $4 \mu \mathrm{g} \mathrm{mL}^{-1}$ monoclonal antibody to myostatin. Error bars denote standard deviation $(n=8)$. Different small letters represent significant difference at $P<0.05$, and different large letters represent significant difference at $P<0.01$.

\subsection{Expression pattern of adipogenic transcription factors}

Adipogenic differentiation induction suppressed myostatin gene expression on day 2 (Figure 4). Exogenous addition of myostatin protein had no effect on endogenous myostatin gene expression in pMDSCs. However, supplemental arginine or myostatin antibody increased myostatin gene expression $(P>0.05)$ and significant promotion was detected in

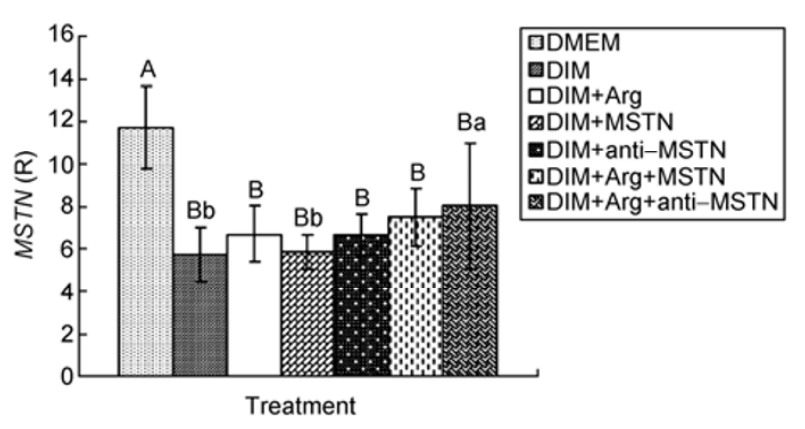

Figure 4 Myostatin gene expression in pMDSCs after $2 \mathrm{~d}$ adipogenic induction. Error bars denote standard deviation $(n=6)$. Different small letters represent significant difference at $P<0.05$, and different large letters represent significant difference at $P<0.01$.

cells treated with a combination of arginine and myostatin antibody $(P<0.05)$. Regarding the adipogenic transcription factors, arginine supplementation promoted $A D D 1$ expression $(P<0.01)$ and increased $C / E B P \alpha, P P A R \delta$ and $L P L$ $(P>0.05)$ in pMDSCs. Exogenous myostatin protein suppressed $P P A R \gamma 2$ and $a P 2$ expression $(P<0.01)$, while myostatin antibody promoted $A D D 1$ expression but suppressed $P P A R \gamma 2$ and $a P 2$ expression $(P<0.05)$. Furthermore, $A D D 1$ and $P P A R \delta$ expression were promoted by a combination of arginine and myostatin, compared to myostatin alone $(P<0.01)$, though no changes in PPAR $\gamma 2$ and $a P 2$ expression were observed. A combination of arginine and myo- 
Table 2 Expression pattern of adipogenic transcriptional factors ${ }^{\text {a) }}$

\begin{tabular}{ccccccccccc}
\hline Treatments & C/EBP $\alpha$ & PPAR 2 & $L P L$ & $a P 2$ & ADD1 & PPAR $\delta$ & GATA-2 & GATA-3 \\
\hline DMEM-HG & $1.00 \pm 0.08^{\mathrm{A}}$ & $1.00 \pm 0.21^{\mathrm{A}}$ & $1.08 \pm 0.25^{\mathrm{ABab}}$ & $3.83 \pm 1.19^{\mathrm{Aa}}$ & $1.00 \pm 0.11^{\mathrm{A}}$ & $1.56 \pm 0.09^{\mathrm{A}}$ & $1.30 \pm 0.10^{\mathrm{Aa}}$ & $1.22 \pm 0.06$ \\
DIM & $1.26 \pm 0.15$ & $6.69 \pm 2.18^{\mathrm{Cd}}$ & $1.44 \pm 0.25^{\mathrm{Bbc}}$ & $12.10 \pm 2.22^{\mathrm{Bc}}$ & $1.24 \pm 0.14^{\mathrm{ABa}}$ & $2.06 \pm 0.42^{\mathrm{AB}}$ & $1.11 \pm 0.19$ & $1.15 \pm 0.2$ \\
DIM+Arg & $1.42 \pm 0.16^{\mathrm{Bb}}$ & $4.99 \pm 2.03^{\mathrm{BCcd}}$ & $1.55 \pm 0.38^{\mathrm{Bc}}$ & $11.66 \pm 4.07^{\mathrm{Bc}}$ & $1.70 \pm 0.23^{\mathrm{C}}$ & $2.33 \pm 0.34^{\mathrm{B}}$ & $1.22 \pm 0.14^{\mathrm{ABabc}}$ & $1.11 \pm 0.14$ \\
DIM+MSTN & $1.08 \pm 0.06^{\mathrm{a}}$ & $2.25 \pm 0.92^{\mathrm{Aa}}$ & $1.27 \pm 0.17^{\mathrm{B}}$ & $4.08 \pm 2.01^{\mathrm{A}}$ & $1.05 \pm 0.13^{\mathrm{A}}$ & $2.31 \pm 0.64^{\mathrm{B}}$ & $1.07 \pm 0.13^{\mathrm{cd}}$ & $1.00 \pm 0.14$ \\
DIM+anti-MSTN & $1.09 \pm 0.09^{\mathrm{a}}$ & $4.63 \pm 1.72^{\mathrm{BCbc}}$ & $1.40 \pm 0.32^{\mathrm{Bbc}}$ & $7.43 \pm 3.67^{\mathrm{b}}$ & $1.53 \pm 0.19^{\mathrm{Bb}}$ & $2.34 \pm 0.45^{\mathrm{B}}$ & $1.00 \pm 0.20^{\mathrm{Bd}}$ & $1.09 \pm 0.08$ \\
DIM+Arg+MSTN & $1.17 \pm 0.08$ & $2.78 \pm 0.59^{\mathrm{ABab}}$ & $1.00 \pm 0.13^{\mathrm{Aa}}$ & $3.97 \pm 1.02^{\mathrm{Aa}}$ & $1.59 \pm 0.21^{\mathrm{BCb}}$ & $3.06 \pm 0.27^{\mathrm{C}}$ & $1.10 \pm 0.10^{\mathrm{bd}}$ & $1.20 \pm 0.11$ \\
DIM+Arg+anti-MSTN & $1.42 \pm 0.52^{\mathrm{Bb}}$ & $6.85 \pm 2.16^{\mathrm{Cd}}$ & $2.29 \pm 0.63^{\mathrm{C}}$ & $9.34 \pm 4.65^{\mathrm{B}}$ & $2.33 \pm 0.50^{\mathrm{D}}$ & $3.35 \pm 0.57^{\mathrm{C}}$ & $1.30 \pm 0.29^{\mathrm{Aab}}$ & $1.30 \pm 0.37$ \\
$P$-value & 0.0093 & $<0.0001$ & $<0.0001$ & $<0.0001$ & $<0.0001$ & $<0.0001$ & 0.0283 & 0.1720 \\
\hline
\end{tabular}

a) The results are shown as means \pm standard deviation, $n=6$. Different small letters following figures in the same column represent significant difference at $P<0.05$, and different capital letters following figures in the same column represent significant difference at $P<0.01$.

statin antibody promoted the expression of $A D D 1, P P A R \delta$, $C / E B P \alpha, P P A R \gamma 2$ and $L P L$ in pMDSCs, compared to myostatin antibody alone $(P<0.05)$. Unexpectedly, the results showed that adipogenic differentiation induction downregulated GATA-2 and GATA-3 expression, and arginine and myostatin treatment had no obvious effects on their expression. These results suggest that myostatin suppressed $P P A R \gamma 2$ and $a P 2$ expression, and that this inhibition could be alleviated by arginine supplementation, at least in part, through promoting $A D D 1$ and $P P A R \delta$ expression in pMDSCs.

\section{Discussion}

Although their precise identification remains a challenge [22], MSCs have been defined as being derived from mesenchymal tissue, with the functional capacities for self-renewal and multipotent differentiation [23]. In accord with several previous studies [24,25], the current study confirmed the mesenchymal nature (CD29-, CD90-positive expression) and non-hematopoietic, non-endothelial origin of the cells, based on their lack of cell surface expression of CD45, CD34 and CD31. The fibroblast-like spindle morphology, cell surface epitopes, and capacity for multilineage mesenchymal differentiation confirmed that the cells used in this study were pMDSCs.

Animal growth performance has recently been improved by genetic selection, comfortable circumstances, and optimal nutrition, though these advances have been at the cost of meat quality [1]. IMF is one of the most important indexes of meat quality, and is positively correlated with factors such as shearing force, tenderness, and flavor. At the cellular level, the IMF content depends on the adipocyte number and the lipid accumulation [26]. Satellite cells or MSCs residing in postnatal muscle can be induced to differentiate into adipocytes [4-7], and are believed to be the origin of IMF. The differentiation of MSCs in skeletal mus- cle into adipocytes leads to increased adipocyte number and lipid accumulation [8], and investigation into the modulation of adipogenic differentiation of muscle-derived MSCs would therefore improve our understanding of the mechanisms of IMF deposition, and allow the exploration of novel approaches to improving the IMF content.

Myostatin is known as a negative regulator of skeletal muscle growth and development, and has recently been reported to be involved in fat deposition and adipogenic differentiation modulation [27,28]. However, the mechanisms whereby myostatin influences adipogenic differentiation are still poorly understood. At the molecular level, adipogenesis results from transcriptional remodeling leading to the activation of numerous genes, including PPAR $2, C / E B P \alpha, L P L$, and $a P 2$ [16]. Several transcription factors act cooperatively and sequentially in this process [26]. At the early stage, adipogenic differentiation is switched on by activation of $A D D 1$ and PPAR $\delta$ [29], and GATA-2 and GATA-3 have been identified as the molecular gatekeepers of that transition. When genes are defective or missing, preadipocytes turn into fat cells, and conversely, high expression levels lead cells to remain as preadipocytes, with little lipid accumulation [30-32]. This study therefore investigated several adipogenic transcription factors, to improve our understanding of the molecular mechanisms involved.

Adipose tissue primarily develops around birth, but adipose cell numbers can increase throughout life in response to nutritional changes [29]. Arginine is a conditionally essential amino acid for pregnant and lactating sows and newborn piglets, and is important for the growth and development of the fetus and neonate [9-11]. A high-fat diet and arginine supplementation were recently reported by Jobgen et al. to differentially regulate gene expression and affect energy-substrate oxidation, redox state, fat accretion, and adipocyte differentiation in adipose tissue, and a beneficial effect of arginine on ameliorating diet-induced obesity in rats was demonstrated [33]. Dietary supplementation with arginine increased muscle gain and decreased subcutaneous 
fat deposition in growing-finishing pigs, and, arginine enhanced longissimus dorsi muscle protein, glycogen, and fat contents by $4.8 \%, 42 \%$, and $70 \%$, respectively [12]. These results indicated that arginine inhibited subcutaneous fat but promoted IMF deposition. However, the modulation of IMF by arginine is still poorly understood. The current study confirmed the inhibition and promotion of adipogenesis in pMDSCs by myostatin and arginine, respectively, and indicated that the inhibitory effect of myostatin on adipogenic differentiation could be alleviated by arginine supplementation. $A D D 1, C / E B P \alpha, P P A R \delta, G A T A-2$ and GATA-3 expression in pMDSCs were up-regulated by adipogenic induction, but arginine and myostatin treatment had no obvious effect on GATA-2 and GATA-3 expression. Our results suggest that myostatin inhibited adipogenesis by suppressing PPAR $\gamma 2$ and $a P 2$ expression in pMDSCs, and that this inhibition could be alleviated by arginine supplementation, at least in part, through promoting $A D D 1$ and PPAR $\delta$ expression. The promotion of adipogenesis in pMDSCs by arginine is in agreement with the results of Tan et al., who reported that dietary arginine supplementation increased the IMF content in growing-finishing pigs [12]. It seems paradoxical that myostatin inhibited adipogenesis, while endogenous myostatin expression was promoted by supplemental arginine in pMDSCs. Nevertheless, our results suggest that myostatin protein and arginine had no significant effects on the numbers of adipogenic cells, and arginine exhibited limited effects on endogenous myostatin expression, suggesting that the modulation of adipogenesis by arginine could not be attributed to endogenous myostatin expression. Further investigations are needed to clarify the molecular mechanisms responsible for the effects of myostatin and arginine on adipogenesis, and to identify novel ways of improving the IMF content in animal production.

In conclusion, the results of this study indicate that adipogenesis is inhibited by the addition of myostatin, and that arginine promotes adipogenic differentiation in pMDSCs. The inhibitory effect of myostatin on adipogenic differentiation can be alleviated by arginine supplementation, at least partially through up-regulation of $A D D 1$ and PPAR $\delta$ expression.

This work was supported by the National Natural Science Foundation of China (Grant No. 30972119).

1 Schwab C R, Baas T J, Stalder K J, et al. Deposition rates and accretion patterns of intramuscular fat, loin muscle area, and backfat of Duroc pigs sired by boars from two time periods. J Anim Sci, 2007, 85: $1540-1546$

2 Schwab C R, Mote B E, Du Z Q, et al. An evaluation of four candidate genes for use in selection programs aimed at increased intramuscular fat in Duroc swine. J Anim Breed Genet, 2009, 126: 228-236

3 Cordero G, Isabel B, Menoyo D, et al. Dietary CLA alters intramuscular fat and fatty acid composition of pig skeletal muscle and subcutaneous adipose tissue. Meat Sci, 2010, 85: 235-239
4 Yada E, Yamanouchi K, Nishihara M. Adipogenic potential of satellite cells from distinct skeletal muscle origins in the rat. J Vet Med Sci, 2006, 68: 479-486

5 Scarda A, Franzin C, Milan G, et al. Increased adipogenic conversion of muscle satellite cells in obese Zucker rats. Int J Obes (Lond), 2010, 34: 1319-1327

6 De Coppi P, Milan G, Scarda A, et al. Rosiglitazone modifies the adipogenic potential of human muscle satellite cells. Diabetologia, 2006, 49 1962-1973

7 Asakura A, Komaki M, Rudnicki M. Muscle satellite cells are multipotential stem cells that exhibit myogenic, osteogenic, and adipogenic differentiation. Differentiation, 2001, 68: 245-253

8 Vettor R, Milan G, Franzin C, et al. The origin of intermuscular adipose tissue and its pathophysiological implications. Am J Physiol Endocrinol Metab, 2009, 297: 987-998

9 Novak S, Moore H S, Paradis F, et al. Temporal candidate gene expression patterns in the sow placenta during early gestation and the effect of maternal L-arginine supplementation. Soc Reprod Fertil Suppl, 2009, 66: 201-202

10 Geng M, Li T, Kong X, et al. Reduced expression of intestinal $\mathrm{N}$-acetylglutamate synthase in suckling piglets: a novel molecular mechanism for arginine as a nutritionally essential amino acid for neonates. Amino Acids, 2010, 40: 1513-1522

11 Wu G, Knabe D A, Kim S W. Arginine nutrition in neonatal pigs. J Nutr, 2004, 134: 2783S-2790S

12 Tan B, Yin Y, Liu Z, et al. Dietary L-arginine supplementation increases muscle gain and reduces body fat mass in growing-finishing pigs. Amino Acids, 2009, 37: 169-175

13 McPherron A C, Lawler A M, Lee S J. Regulation of skeletal muscle mass in mice by a new TGF-beta superfamily member. Nature, 1997, 387: 83-90

14 McPherron A C, Lee S J. Double muscling in cattle due to mutations in the myostatin gene. Proc Natl Acad Sci USA, 1997, 94: 1245712461

15 Artaza J N, Bhasin S, Magee T R, et al. Myostatin inhibits myogenesis and promotes adipogenesis in 10T (1/2) mesenchymal multi-potent cells. Endocrinology, 2005, 146: 3547-3557

16 Feldman B J, Streeper R S, Farese R V, et al. Myostatin modulates adipogenesis to generate adipocytes with favorable metabolic effects. Proc Natl Acad Sci USA, 2006, 103: 15675-15680

17 Guo W, Flanagan J, Jasuja R, et al. The effects of myostatin on adipogenic differentiation of human bone marrow-derived mesenchymal stem cells are mediated through cross-communication between Smad3 and Wnt/beta-catenin signaling pathways. J Biol Chem, 2008, 283: 9136-9145

18 Sheyn D, Pelled G, Zilberman Y, et al. Nonvirally engineered porcine adipose tissue-derived stem cells: use in posterior spinal fusion. Stem Cells, 2008, 26: 1056-1064

19 Guillot P V, Gotherstrom C, Chana J, et al. Human first-trimester fetal MSC express pluripotency markers and grow faster and have longer telomeres than adult MSC. Stem cells, 2007, 25: 646-654

20 Baksh D, Yao R, Tuan R S. Comparison of proliferative and multilineage differentiation potential of human mesenchymal stem cells derived from umbilical cord and bone marrow. Stem cells, 2007, 25: 1384-1392

21 Vandesompele J, De Preter K, Pattyn F, et al. Accurate normalization of real-time quantitative RT-PCR data by geometric averaging of multiple internal control genes. Genome Biol, 2002, 3: 1-12

22 Baksh D, Song L, Tuan R S. Adult mesenchymal stem cells: characterization, differentiation, and application in cell and gene therapy. $\mathrm{J}$ Cell Mol Med, 2004, 8: 301-316

23 McKay R. Stem cells in the central nervous system. Science, 1997, 276: 66-71

24 Gang E J, Bosnakovski D, Figueiredo C A, et al. SSEA-4 identifies mesenchymal stem cells from bone marrow. Blood, 2007, 109: 17431751

25 Case J, Horvath T L, Ballas C B, et al. In vitro clonal analysis of murine pluripotent stem cells isolated from skeletal muscle and adipose stromal cells. Exp Hematol, 2008, 36: 224-234 
26 Niemela S M, Miettinen S, Konttinen Y, et al. Fat tissue: views on reconstruction and exploitation. J Craniofac Surg, 2007, 18: 325-335

27 McPherron A C, Lee S J. Suppression of body fat accumulation in myostatin-deficient mice. J Clin Invest, 2002, 109: 595-601

28 Hirai S, Matsumoto H, Hino N, et al. Myostatin inhibits differentiation of bovine preadipocyte. Domest Anim Endocrin, 2007, 32: 1-14

29 Grimaldi P A. The roles of PPARs in adipocyte differentiation. Prog Lipid Res, 2001, 40: 269-281

30 Tong Q, Dalgin G, Xu H, et al. Function of GATA transcription factors in preadipocyte-adipocyte transition. Science, 2000, 290: 134-138

31 Tong Q, Tsai J, Hotamisligil G S. GATA transcription factors and fat cell formation. Drug News Perspect, 2003, 16: 585-588

32 Tsai J, Tong Q, Tan G, et al. The transcription factor GATA2 regulates differentiation of brown adipocytes. EMBO Rep, 2005, 6: 879-884

33 Jobgen $\mathrm{W}, \mathrm{Fu} \mathrm{W}$, Gao $\mathrm{H}$, et al. High fat feeding and dietary L-arginine supplementation differentially regulate gene expression in rat white adipose tissue. Amino Acids, 2009, 37: 187-198

Open Access This article is distributed under the terms of the Creative Commons Attribution License which permits any use, distribution, and reproduction in any medium, provided the original author(s) and source are credited. 